\title{
T TAURI AND FU ORIONIS STARS
}

\author{
G. F. GAHM \\ Stockholm Observatory, Saltsjöbaden, Sweden
}

\begin{abstract}
A great deal of information on the continuous radiation of T Tau- and similar type stars has been acquired. The cause of the ultraviolet and infrared excess emission is discussed. The possibility that the blue continuum may arise from numerous line emissions is considered. Various possibilities for the cause of the light variations are described, as well as the nature of the emission lines. The special features of the FU Ori stars are mentioned.
\end{abstract}

\section{Introduction}

FU Ori entered the scene in 1936/37. The remarkable flare-up was interpreted by Herbig (1966) as the result of a rapid evolutionary transition of a pre-main-sequence object. In 1969/70, $\mathrm{LKH}_{\alpha} 190=\mathrm{V} 1057 \mathrm{Cyg}$ took its turn. The flare-up was discovered by Welin (1971).

The photometric behaviour and the post-outburst spectra of these stars are very similar, and there is no reason to doubt that the flare-ups are manifestations of the same phenomenon. In the case of V 1057 Cyg we know that the pre-outburst spectrum was that of a T Tauri star (Herbig, 1958). Even though it is difficult to make statistics on two stars, it now appears that the FU Ori-phenomenon is not an extremely rare one and that perhaps a not-negligible fraction of the $T$ Tauri stars goes through such a phase. Obviously we must understand the nature of $\mathrm{T}$ Tauri stars in order to understand the FU Ori-stars, and in the following I will discuss certain aspects of the continuous energy distribution, the cause of the light-variations and the nature of the lineemitting region of $\mathrm{T}$ Tauri stars before returning to the FU Ori stars for a brief comment.

The T Tauri-group of stars was defined by Herbig (1962) primarily on the basis of certain spectral characteristics as observed in the optical spectral region. I will follow this scheme although other objects which, like the T Tauri stars, are believed to be in their pre-main-sequence phase of evolution may enter the discussion on occasion.

\section{The Continuous Energy Distribution of T Tauri Stars}

In order to establish the continuous energy distribution of T Tauri stars it is necessary to observe over a large spectral range, preferably in narrow bands with photoelectric techniques. Care must be taken to avoid or correct for emission lines and to check for variability of stellar light during the observations. Many $\mathrm{T}$ Tauri stars are surrounded by extended regions of bright nebulosity which contribute in the measured flux. In the last years a great deal of information on energy distributions of $T$ Tauri stars have appeared. Several stars have been surveyed with a photoelectric spectrum scanner 
from $\lambda 3300$ to $\lambda 11000$ by Kuhi (1974). Infrared photoelectric observations by Mendoza (1966, 1968), Low et al. (1970), Geisel (1970), Lee (1970) and Cohen (1973) have extended the observed spectral range into the $20 \mu$ region. Radio emission at 3.7 and $11 \mathrm{~cm}$ from T Tau and $\mathrm{LkH} \alpha-101$ has been reported by Spencer and Schwartz (1974).

The presence of an ultraviolet excess emission in $\mathrm{T}$ Tauri stars was established long ago by Haro (Haro and Herbig, 1955) and Walker (1956) and some stars have for a long time been known to exhibit a continuous excess emission in the blue (Herbig, 1958). In addition, the infrared observations have revealed a continuous infrared excess emission. It was shown by Kuhi (1974) that the fluxes at $\lambda 3620$ and in the emission lines of $\mathrm{H} \alpha$ and $\mathrm{Ca}$ II $\mathrm{K}$ are coupled. It seems clear that a large fraction, if not all, of the ultraviolet excess originates from hydrogen free-bound emission in an emitting region round the star and that much of the line emission comes from the same region. In such a region additional continuous emission over the optical and infrared domains can be expected. In fact, Strom (1972) has argued that the infrared excess of T Tauri stars is the result of hydrogen free-free emission. The evidence comes from the fact that the $3.5 \mu$ flux seems to follow the $\mathrm{H} \alpha$ and $\lambda 3620$ fluxes. A completely different interpretation is that the infrared excess arises from thermal reradiation from circumstellar dust (Low and Smith, 1966). Several indications of circumstellar dust round $\mathrm{T}$ Tauri stars exist, some of which will be discussed in the next section. In a statistical study by Götz (1965) it was found that stars with strong $\mathrm{H} \alpha$ emission tend to be situated in cloud regions of higher obscuration than stars with weak $\mathrm{H} \alpha$ emission. It is possible that the $\mathrm{H} \alpha$ flux and the density of dust close to the star go hand in hand giving the correlations discussed by Strom. It seems to me likely that both dust emission and hydrogen free-free emission contribute in the infrared.

The nature of the blue continuum is not clear. It is often described as a veiling or a complete obliteration of the ordinary absorption spectrum. This indicates that the origin of the excess is not photospheric. The possibility that the blue continuum is the composite contribution of a great number of faint emission lines was discussed by Herbig (1958). In general the smoothness of the continuum is taken as evidence against such an interpretation. The presence of a nearly continuous mat of weak, unresolved emission lines was recently established on RU Lup by Gahm et al. (1974) who note that there are only narrow spectral regions where line emission is negligible. The main contribution comes from Fe I lines. Longward of $4500 \AA$ this line background is unimportant. The weak and strong lines are formed in the same region. Other stars with advanced $\mathrm{T}$ Tauri-type spectra most probably have similar background emission. For these stars it seems very likely that a considerable fraction of the blue excess continuum is explained in this way.

\section{The Cause of the Light Variations}

The light variations on $\mathrm{T}$ Tauri stars are in some cases very dramatic. A star may change its visual brightness by a factor of 2 or more over one night, and sometimes detectable changes occur on a time-scale of minutes. It has been noted by several investiga- 
tors that the stars in general become redder with decreasing brightness (see e.g. Brück, 1971; Nandy and Pratt, 1972). This is not always the case, however. For instance Badaljan (1958) found from studies of a large number of $T$ Tauri stars that the amplitudes of the photographic magnitudes can be larger than, approximately equal to or even smaller than the corresponding photovisual amplitudes. It is sometimes difficult to judge how the emission lines effect such variations. Very few simultaneous spectroscopic and photoelectric observations have been carried out with the purpose of investigating the possible interplay between variations in intensities, widths and profiles of emission lines with variations in different continua. Some information of this kind is available for RW Aur (Kharadze and Bartaya, 1965; Gahm, 1970; Chalonge et al., 1971) and for DI Cep (Petrov, 1972) indicating a close relationship between the physical properties in the line-emitting region and the stellar continuum changes. In the light of the extremely rapid spectral changes reported (e.g. Weston and Aller, 1955; Ismailov, 1972, 1973), the idea of flare-phenomena on the stars as the cause of light and spectral variations is not far-fetched. Several models introduce the concept of flare activity. In the view of Ambartsumian (1971), concentrated energy sources of an unknown nature are released in the atmospheres. Effects of the release of fast electrons from active regions have been discussed by Gordon (1958) and Gurzadyan (1965, 1970, 1973). Kurochkin (1963) interpreted the characteristic time-scale for the light variations of $T$ Ori in terms of the characteristic time for convective cells to spread on the surface.

However, for some stars a completely different cause of the light variations seems to apply. Anderson and Kuhi (1968) and Kuhi (1969) noticed that part of the relative intensity variations in the lower Balmer lines could be the result of variable circumstellar extinction. Wenzel $(1969,1970,1971)$ found from UBV measures that SV Cep and RY Tau pass through sharp minima on a time-scale of days without any signifcant colour changes. These observations were interpreted in terms of time-variable, gray, circumstellar absorption. The character and time-scale of the variations seem to imply that a large number of dust concentrations of stellar dimensions move close to the star and occasionally occult it. A strong time-variable degree of polarization was discovered in R Mon by Zellner (1970) and in RY Tau by Breger (1974), results which point to the same general conclusion of variable circumstellar extinction. Strong evidence of variable circumstellar extinction as the cause of the light variations on RU Lup was found by Gahm et al. (1974). The evidence comes from the fact that the amplitude of the light variations follows a wavelength dependence similar to the absorption of interstellar dust and that the spectral properties of the star remained practically constant over the period of observations. In particular the ratio of the intensity of emission lines to that of the underlying continuum is practically constant over nights when the star as a whole changes its brightness by up to $60 \%$ in the blue. Again, the interpretation is that of dust concentrations of stellar dimensions moving close to the star.

It therefore seems that even though a large number of $T$ Tauri stars vary because of large-scale changes in the photospheric and line-emitting regions there are also super- 
imposed variations of circumstellar origin. Maybe the variations on certain stars are exclusively of extrinsic nature. Furthermore, a new and interesting aspect of the circumstellar surroundings takes form. Protoplanets have been discussed in astronomy for a long time. Maybe, we are now starting to see characteristic dimensions in such systems. Still we do not know whether these dust concentrations, or dust streams, are examples of protoplanets or just local irregularities in a dust envelope, which in a later phase will be blown away.

\section{Comments on the Nature of the Line-Emitting Region}

As noted by Joy (1945) the emission line spectra of 'advanced' T Tauri stars are reminiscent of the solar chromospheric spectrum. The spectral state of the lineemitting region indicates that this region in general is hotter than the underlying star. The source of excitation of this region is not established. Possible mechanisms have been reviewed by Bodenheimer (1972). An outline of a model in which the temperature inversion would be the result of a mechanical energy transport penetrating out to the thinner surroundings where energy is deposited has been given by Herbig (1970). This is an attractive idea since we know that this happens on the Sun. Furthermore the $T$ Tauri stars can be identified with low mass stars having sizable outer convection zones in the model computations by Larson (1972) and Hayashi (1970).

Broad, red-displaced absorption components are present on a group of $\mathbf{T}$ Taurilike stars investigated by Walker (1972). Many T Tauri stars, however, show violetdisplaced absorption features superimposed on the strongest emission lines. This led Kuhi $(1964,1966)$ to define a model in which material is decelerated in the gravitational field of the star after being ejected radially outwards. Computed line-profiles were fitted to observed profiles resulting in extended emitting volumes. It was later shown by Dumont et al. (1973) that the observed emission features can be produced without recourse to an extended atmosphere. In fact, the line-emitting region may be quite small, similar to a chromosphere. Computations of the effects of supersonic turbulent motions in the outer convective regions of $\mathrm{T}$ Tauri stars have been carried out by Prentice (1973), who discussed the possibility that the mass-ejection phenomenon results from supersonic convective overshooting in the stable atmosphere of the star. In this model no mass leaves the star. On the assumption that the mechanical flux from stars is distributed in turbulent eddies of supersonic velocities, Scharmer (1974) recently found a theoretical explanation of the Wilson-Bappu effect. He applied this theory to the T Tauri stars and found that if the emission line profiles are controlled by supersonic turbulence, the mechanical flux required is of the order of $10^{-2}$ of the radiative flux from the photosphere for stars with weak emission and of the order of unity for 'advanced' types. This model gives automatically a mass loss driven by the supersonic turbulence.

Could it be that the line and continuous emission we observe from $T$ Tauri stars arise in a thin chromospheric region close to the star as the result of the presence of a 
strong mechanical flux generated in the deep convective layers, and that the extreme widths of the emission lines is the result of supersonic mass motions, perhaps of turbulent nature, in this chromosphere? As noted by Herbig (1952) the underlying absorption spectra of $\mathrm{T}$ Tauri stars often show abnormally broad and diffuse absorption lines. The widths of these lines were expressed in $v \sin i / s$ of $\sim 50 \mathrm{~km} \mathrm{~s}^{-1}$. As remarked by Herbig, the broadening agent of the lines may also be large-scale turbulence. Such a turbulence is clearly supersonic.

If we could point at one $\mathrm{T}$ Tauri star for which the light variations are of exclusively extrinsic nature, the blue continuum is composed of emission lines and the emission lines are formed in a chromosphere, maybe, this star would look rather attractive to people working with model stellar atmospheres. All efforts to construct models and alternative explanations for the outer layers of $\mathrm{T}$ Tauri stars should be encouraged.

\section{The FU Ori Stars}

Three different, but not necessarily mutually independent interpretations of the FU Ori phenomenon can be visualized. First, one could argue that during the outbursts a reorganization of energy sources took place. This argument is implicit in Ambartsumian's (1971) hypothesis where, in his terminology, the FUORS are manifestations of the release of energy from non-thermal sources. A transfer of particle-flux luminosity to radiative luminosity would lead to an increase in observed flux, but this latter explanation does not seem sufficient to account for the enormous increase in optical brightness.

Secondly, the stars may have gone through a rapid evolutionary transition as discussed by e.g. Pişmiş (1971) and Grasdalen (1973). This explanation seems to offer some difficulties from the theoretical point of view, mainly because of the short timescale involved. The first and second interpretations require an intrinsic change of the stellar object. Support for this conclusion comes from the change in spectral type and colour that has been reported to take place on V 1057 Cyg after the outburst (e.g. Gieseking, 1974).

Finally, the clearing of a circumstellar dust shell as the cause of the flare-up at optical wavelengths has been discussed by Cohen and Woolf (1971) and Larson (1972). The post-outburst decrease of V $1057 \mathrm{Cyg}$ in the $11 \mu$ and $20 \mu$ regions, where the 'silicate signature' is noticeable, was demonstrated by Simon et al. (1972) from comparisons with the observations by Cohen and Woolf. A decrease in the $1720 \mathrm{MHz} O H$ emission by a factor 5 over 5 months was found by Lo and Bechis (1973) and Lo and Lo (1974). There is no evidence for $\mathrm{OH}$ emission from FU Ori at the four main $\mathrm{OH}$ frequencies (Gahm and Winnberg, 1971) and the $10 \mu$ flux from this star is weaker than for V 1057 Cyg (Cohen and Woolf, 1971). It could be argued that these observations support the third hypothesis. Of course, a clearing of a dust envelope may require help from inside. If we adopt the view that a large fraction of the $T$ Tauri stars go through the FU Ori-phase as a part of their evolution, then we must admit that an intrinsic change of these stars takes place. 


\section{References}

Anderson, L. and Kuhi, L. V.: 1968, in L. Detre (ed.), Non-Periodic Phenomena in Variable Stars,

Reidel, Dordrecht, p. 93.

Ambartsumian, V. A.: 1971, Astrofizika 7, 557.

Badaljan, G. S.: 1962, Contr. Burakan Obs. 31, 57.

Bodenheimer, P. 1972, Rep. Prog. Phys. 35, 1.

Breger, M.: 1974, Astrophys. J. 188, 53.

Brück, M. T.: 1971, Publ. Roy. Obs. Edinburgh 7, 63.

Chalonge, D., Divan, L., and Mirzoyan, L. V.: 1971, Astrofizika 7, 345.

Cohen, M.: 1973, Monthly Notices Roy. Astron. Soc. 161, 97.

Cohen, M. and Woolf, N. J.: 1971, Astrophys. J. 169, 543.

Dumont, S., Heideman, N., Kuhi, L. V. and Thomas, R. N.: 1973, Astron. Astrophys. 29, 199.

Gahm, G. F.: 1970, Astron. Astrophys. 8, 73.

Gahm, G. F. and Winnberg, A.: 1971, Astron. Astrophys. 13, 489.

Gahm, G. F., Nordh, H. L., Olofsson, S. G., and Carlborg, N. C. J.: 1974, Astron. Astrophys. 33, 399.

Geisel, S. L.: 1970, Astrophys. J. 161, L105.

Gieseking, F.: 1974, Astron. Astrophys. 31, 117.

Götz, W.: 1965, Veröff. Sternwarte Sonneberg 7, 1.

Gordon, I. M.: 1958, Astron. Zh. USSR 35, 458.

Grasdalen, G. L. : 1973, Astrophys. J. 182, 781.

Gurzadyan, G. A.: 1965, Astrofizika 1, 319.

Gurzadyan, G. A.: 1970, Bol. Obs. Tonantzintla Tacubaya 5, 225.

Gurzadyan, G. A.: 1973, Astron: Astrophys. $28,147$.

Haro, G. and Herbig, G. H.: 1955, Bol. Obs. Tonantzintla Tacubaya No. 12, 33.

Hayashi, C.: 1970, Mem. Soc. Roy. Sci. Liège, 5th Series 19, 127.

Herbig, G. H.: 1952, J. Roy. Astron. Soc. Canada 46, 222.

Herbig, G. H.: 1958, Mem. Soc. Roy. Liege, 4th Series 20, 251.

Herbig, G. H. : 1958, Astrophys. J. 128, 259.

Herbig, G. H. : 1962, Adv. Astron. Astrophys. 1, 47.

Herbig, G. H. : 1966, Vistas in Astronomy 8, 109.

Herbig, G. H. : 1970, Mem. Soc. Roy. Sci. Liège, 5th Series 19, 13.

Ismailov, Z. A.: 1972, Astron. Circ., Moscow, No. 734.

Ismailov, Z. A.: 1973, Astron. Circ., Moscow, No. 763.

Joy, A. H. : 1945, Astrophys. J. 102, 207.

Kharadze, E. K. and Bartaya, R. A.: 1964, Bull. Astrophys. Obs. Abastumani No. 30, 3.

Kuhi, L. V.: 1964, Astrophys. J. 140, 1409.

Kuhi, L. V.: 1966, Astrophys. J. 143, 991.

Kuhi, L. V.: 1969, in S. S. Kumar (ed.), Low Luminosity Stars, Gordon and Breach, New York, p. 149.

Kuhi, L. V.: 1974, Astron. Astrophys. Suppl. 15, 47.

Kurochin, N. E.: 1963, Peremennye Zvezdy 14, 284.

Larson, R. B.: 1972, Monthly Notices Roy. Astron. Soc. 157, 121.

Lee, T. A.: 1970, Publ. Astron. Soc. Pacific 82, 765.

Lo, K. Y. and Bechis, K. P.: 1973, Astrophys. J. 185, L71.

Lo, K. Y. and Lo, K. P.: 1974, Bull. Am. Astron. Soc. 6, 22.

Low, F. J., Johnson, H. L., Kleinmann, O. E., Latham, A. S., and Geisel, S. L.: 1970, Astrophys. J. $160,531$.

Low, F. J. and Smith, B. J.: 1966, Nature 212, 675.

Nandy, K. and Pratt, N.: 1972, Astrophys. Space Sci. 19, 219.

Mendoza, E. E.: 1966, Astrophys. J. 143, 1010.

Mendoza, E. E.: 1968, Astrophys. J. 151, 977.

Petrov, P. P.: 1972, Izv. Krymsk. Astrofiz. Obs. 46, 25.

Pişmiş, P.: 1971, Bol. Obs. Tonantzintla Tacubaya 6, 131.

Prentice, A. J. R.: 1973, Astron. Astrophys. 27, 237.

Scharmer, G. : 1974, Astron. Astrophys., in press. 
Simon, T., Morrison, N. D., Wolff, S. C., and Morrison, D.: 1972, Astron. Astrophys. 20, 99.

Spencer, J. H. and Schwartz, P. R.: 1974, Astrophys. J. 188, L105.

Strom, S. E.: 1972, Publ. Astron. Soc. Pacific 84, 745.

Walker, M. F.: 1956, Astrophys. J. Suppl. 2, 365.

Walker, M. F.: 1972, Astrophys. J. 175, 89.

Welin, G.: 1971, Astron. Astrophys. 12, 312.

Wenzel, W.: 1969, Mitt. Veränd. Sterne Sonneberg 5, 75.

Wenzel, W.: 1970, Mitt. Veränd. Sterne Sonneberg 5, 117.

Wenzel, W.: 1971, Astron. Nachr. 292, 221.

Weston, E. B. and Aller, L. H.: 1955, Mem. Soc. Roy. Sci. Liège, 4th Series 15, 251.

Zellner, B.: 1970, Astron. J. 75, 182. 